\title{
TRANSIÇÃO DO CUIDADO DO HOSPITAL PARA O DOMICÍLIO: REVISÃO INTEGRATIVA
}

\author{
Luciana Andressa Feil Weber ${ }^{1}$, Maria Alice Dias da Silva Lima², Aline Marques Acosta ${ }^{3}$, Giselda Quintana \\ Maques $^{4}$
}

RESUMO: Objetivou-se identificar atividades dos enfermeiros na transição do cuidado do hospital para o domicílio a partir de evidências na literatura. Trata-se de revisão integrativa, realizada em agosto de 2015, mediante busca nas bases de dados PubMed, Cumulative Index to Nursing and Allied Health Literature, Web of Science, SCOPUS e Literatura Latino-Americana e do Caribe em Ciências da Saúde. Foram selecionados 22 artigos que atenderam aos critérios de inclusão. Identificaram-se estudos de intervenção, pesquisas experimentais, quaseexperimentais, em idosos e com doenças crônicas. Os resultados evidenciaram cinco categorias temáticas com as principais atividades dos enfermeiros na transição do cuidado: planejamento de cuidados para a alta; auxílio na reabilitação social; educação em saúde; articulação com os demais serviços; acompanhamento pós-alta. Conclui-se que há necessidade de aprimoramento das práticas assistenciais e organização das atividades dos enfermeiros, promovendo coordenação do cuidado com foco na transição do hospital para o domicílio.

DESCRITORES: Cuidado transicional; Alta do paciente; Continuidade da assistência ao paciente; Enfermagem.

\section{CARE TRANSITION FROM HOSPITAL TO HOME: INTEGRATIVE REVIEW}

\begin{abstract}
The present study aimed to identify nurses' activities in the transition of care from hospital to home based on literature evidence. Integrative review conducted in August 2015, through search of PubMed, Cumulative Index to Nursing and Allied Health Literature, Web of Science, SCOPUS and Latin American and Caribbean Health Sciences Literature databases. Twenty-two (22) articles that met the inclusion criteria were selected. Interventional, experimental and quasi-experimental studies with elderly and patients with chronic diseases were identified. The results showed five thematic categories related to the main activities of nurses in transitional care: planning for discharge; assistance in social rehabilitation; health education; coordination with other services; postdischarge follow-up. It is concluded that improvement of care practices and organization of nurses' activities is needed, to ensure the coordination of care focused on transition from hospital to home.

DESCRIPTORS: Transitional care; Patient discharge; Continuity of Patient Care; Nursing.
\end{abstract}

\section{TRANSICIÓN DEL CUIDADO DEL HOSPITAL PARA EL DOMICILIO: REVISIÓN INTEGRATIVA}

RESUMEN: Estudio cuya finalidad fue identificar actividades de los enfermeros en la transición del cuidado del hospital para el domicilio considerándose evidencias en la literatura. Es una revisión integrativa, realizada en agosto de 2015, por medio de búsqueda en las bases de datos PubMed, Cumulative Index to Nursing and Allied Health Literature, Web of Science, SCOPUS y Literatura Latinoamericana y de Caribe en Ciencias de la Salud. Se seleccionaron 22 artículos de acuerdo a los criterios de inclusión. Se identificaron estudios de intervención, investigaciones experimentales, casi experimentales, en ancianos y con enfermedades crónicas. Los resultados apuntaron cinco categorías temáticas con las principales actividades de los enfermeros en la transición del cuidado: planeamiento de cuidados para el alta; ayuda en la rehabilitación social; educación en salud; articulación con otros servicios; acompañamiento despues del alta. Se constata que hay necesidad de perfeccionamiento de las prácticas asistenciales y organización de las actividades de los enfermeros, para promover coordinación del cuidado con énfasis en la transición del hospital para el domicilio.

DESCRIPTORES: Cuidado transicional; Alta del paciente; Continuidad de la asistencia al paciente; Enfermería.

\footnotetext{
${ }^{1}$ Enfermeira. Mestranda em Enfermagem na Universidade Federal do Rio Grande do Sul. Porto Alegre, RS, Brasil. ${ }^{2}$ Enfermeira. Doutora em Enfermagem. Docente do Programa de Pós-Graduação em Enfermagem da Universidade Federal do Rio Grande do Sul. Porto Alegre, RS, Brasil.

${ }^{3}$ Enfermeira. Doutoranda em Enfermagem na Universidade Federal do Rio Grande do Sul. Porto Alegre, RS, Brasil. ${ }^{4}$ Enfermeira. Doutora em Enfermagem. Estágio Pós-Doutoral em Enfermagem no Programa de Pós-Graduação em Enfermagem da Universidade Federal do Rio Grande do Sul. Porto Alegre, RS, Brasil.
}

Autor Correspondente:

Luciana Andressa Feil Weber

Universidade Federal do Rio Grande do Sul

R. São Manoel, 963 - 90620-110 - Porto Alegre, RS, Brasil

E-mail: luhandressa@gmail.com
Recebido: $07 / 07 / 2016$

Finalizado: 30/06/2017 


\section{INTRODUÇÃO}

A transição do cuidado refere-se a ações para assegurar a coordenação e a continuidade da assistência à saúde, na transferência de pacientes entre diferentes serviços de saúde ou diferentes unidades de um mesmo local ${ }^{(1)}$. Devido ao envelhecimento populacional, crescente prevalência de doenças crônicas, tendências de redução do tempo de permanência hospitalar e do aumento da atenção na comunidade, a transição tem sido destacada como uma das formas de superar a fragmentação da atenção e garantir a continuidade dos cuidados ${ }^{(2)}$.

A transição ocorre em contexto que inclui o paciente, seus familiares e cuidadores, os profissionais que prestaram atendimento e os que continuarão a assistência ${ }^{(1)}$. Portanto, é um processo complexo que exige coordenação e comunicação entre pessoas de diferentes formações, experiências e habilidades.

A alta hospitalar é um momento de mudanças no cotidiano dos pacientes, no qual há acréscimo de medicações e cuidados no domicílio. Essas mudanças, por vezes, não são abordadas de modo eficaz durante a internação hospitalar, proporcionando fragmentação dos cuidados pós-alta ${ }^{(3)}$. É momento que requer planejamento, preparação e educação em saúde do paciente e da família, principalmente de idosos e pessoas com doenças crônicas, que têm necessidades de saúde persistentes e contínuas ${ }^{(1)}$. Porém, as orientações de alta muitas vezes são realizadas de forma mecânica e apressada, sem considerar as condições e as necessidades de cada paciente ${ }^{(4)}$, frequentemente fornecidas apenas no momento da saída do hospital ${ }^{(5)}$.

Mesmo pacientes que se sentem preparados no momento da alta ${ }^{(6)}$, quando retornam para casa, se deparam com incertezas acerca do tratamento e da recuperação ${ }^{(7)}$. A falta ou insuficiência de planejamento de alta pode trazer repercussões que incluem angústia e ansiedade ${ }^{(2)}$, efeitos adversos e erros de medicação ${ }^{(8)}$, pouca aderência ao tratamento e baixa qualidade de $\operatorname{vida}^{(9)}$.

Processos de transição adequados podem melhorar a qualidade dos resultados assistenciais e influenciar a qualidade de vida dos pacientes, contribuindo para evitar reinternações hospitalares desnecessárias e reduzir custos dos cuidados de saúde ${ }^{(5,10)}$. Os enfermeiros têm responsabilidade de assegurar que os pacientes voltem para casa adequadamente preparados e com apoio adequado, contribuindo para melhor articulação e comunicação entre profissionais, pacientes, cuidadores e serviços de saúde, visando proporcionar continuidade do cuidado para estratégias de transição bem sucedidas $^{(11)}$.

Muitas vezes, devido às inúmeras atividades, os enfermeiros fornecem informações apenas durante atividades rotineiras da sua prática assistencial ${ }^{(2)}$, por vezes de modo acelerado e com excesso de orientações em curto período de tempo ${ }^{(12)}$. Além disso, relatam a existência de intensa rotatividade dos leitos hospitalares, que prejudica a transmissão das informações sobre os cuidados domiciliares durante o processo de alta $^{(2)}$.

Os enfermeiros podem ter papel ativo em estratégias de transição do cuidado por meio de sua coordenação, bem como oferecendo suporte de educação em saúde e orientações para a prevenção, controle da doença, promoção e manutenção da saúde ${ }^{(5)}$, a fim de aprimorar o preparo de alta e garantir a continuidade dos cuidados no domicílio. Esse processo está em construção e adaptação em diferentes países e carece de investigação, especialmente no Brasil.

Atualmente a qualidade das transições do cuidado tem sido utilizada como um dos componentes para avaliação de desempenho de hospitais. A garantia de transições seguras e eficientes de serviços hospitalares para o domicílio tem sido foco de interesse de pesquisadores e gestores de saúde no âmbito internacional ${ }^{(5,10)}$. Sendo assim, este artigo tem por objetivo identificar as atividades dos enfermeiros na transição do cuidado na alta do hospital para o domicílio, a partir de evidências na literatura.

\section{- MÉTODO}

Trata-se de revisão integrativa, que seguiu cinco etapas preconizadas para sua execução: identificação do problema, busca na literatura, avaliação dos dados, análise dos dados, e apresentação da síntese do 
conhecimento $^{(13)}$.

A questão norteadora foi: quais são as atividades dos enfermeiros na transição do cuidado do hospital para o domicílio descritas na literatura?

Foi realizada busca nas bases de dados PubMed, Cumulative Índex to Nursing and Allied Health Literature (CINAHL), Web of Science Overview (SCOPUS) e Literatura Latino-Americana e do Caribe em Ciências da Saúde (LILACS).

Para a busca nas bases de dados, utilizaram-se os descritores controlados contidos nos Descritores em Ciências da Saúde (DeCS) e também descritores não controlados, todos em inglês. Foram realizadas as seguintes combinações: 1) (patient discharge) AND (care transitions) AND (best practices) AND (nursing); 2) ((care coordination) OR (care transition)) AND (patient discharge) AND (nursing); 3) ((care coordination) OR (care transition)) AND (patient discharge) AND (nursing) AND (best practices); 4) ((care coordination) OR (care transition) OR (patient discharge)) AND (nursing) AND (best practices).

Os critérios de inclusão foram artigos completos e disponíveis na íntegra, nos idiomas inglês, espanhol e português, publicados no período de janeiro de 2005 a agosto de 2015. Foram excluídos artigos teóricos, revisões integrativas, narrativas e sistemáticas, relatos de experiência, editoriais, teses, dissertações, monografias, resumos, documentos e anais de eventos. A coleta ocorreu no mês de agosto de 2015.

Foram encontrados 2.279 artigos e, após a remoção dos artigos duplicados, totalizaram 1.419 publicações, das quais 105 foram pré-selecionados pela leitura de títulos e resumos. Após leitura minuciosa dos textos, a amostra final foi composta por 22 artigos, conforme apresentado na Figura 1.

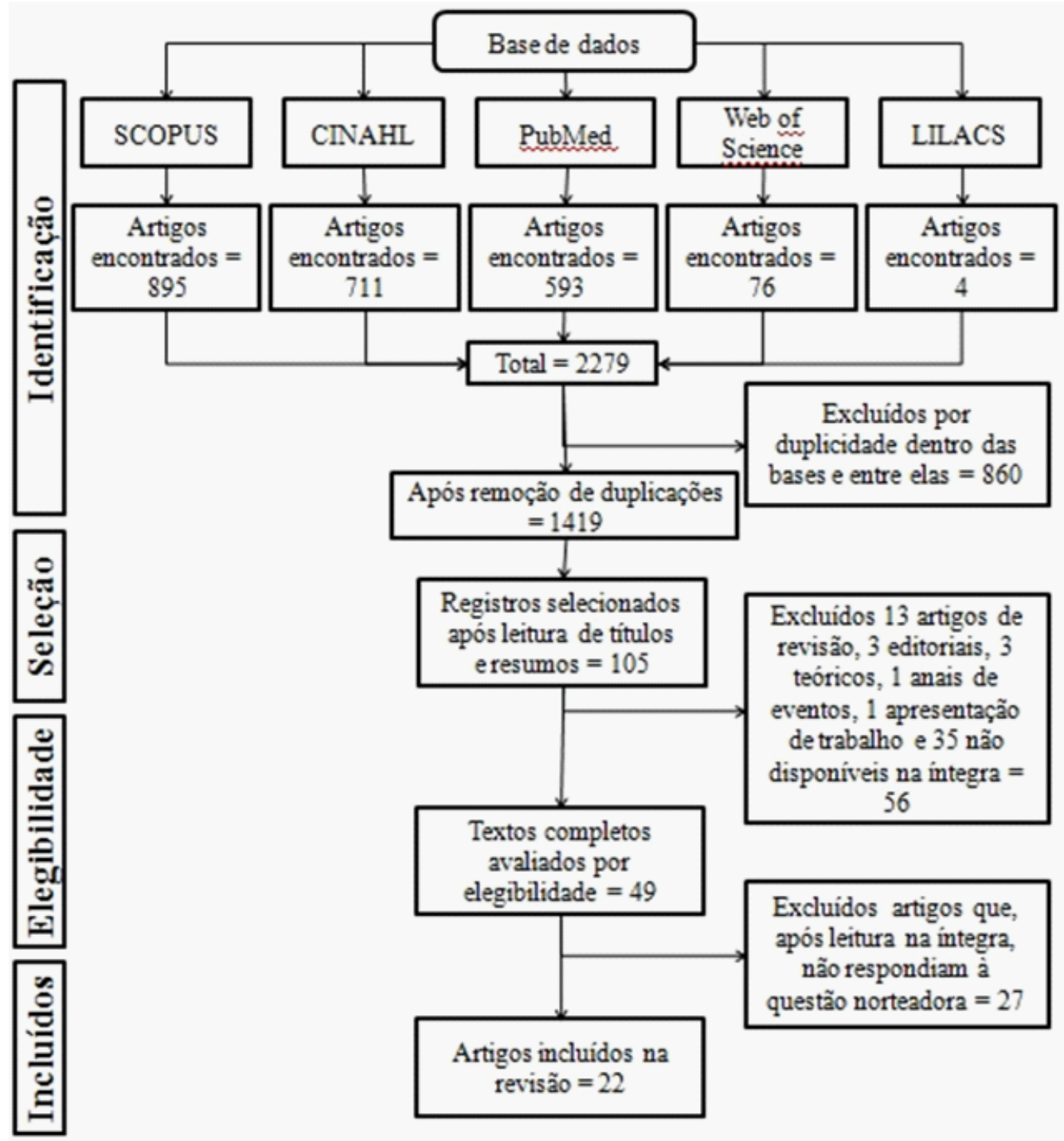

Figura 1 - Fluxograma da coleta de dados e seleção dos estudos que compõem a amostra. Porto Alegre, 2015 
A extração dos dados foi realizada por meio de roteiro com informações sobre ano de publicação, autores, título, periódico, país, delineamento metodológico, objetivos, resultados, atividades dos enfermeiros e conclusões. Para organização dos artigos e remoção das duplicações foi utilizado o gestor de referências EndNote X7.

A análise dos dados foi realizada por meio de cálculos de frequência simples e relativa. As atividades dos enfermeiros descritas nos artigos foram classificadas por similaridade e agrupadas em categorias, com utilização do programa NVivo, versão 10.

\section{RESULTADOS}

A amostra desta revisão foi composta por 22 estudos que descrevem atividades ou propõem intervenções a serem realizadas pelos enfermeiros, com a finalidade de qualificar a transição na alta do hospital para o domicílio. O Quadro 1 apresenta os artigos, conforme autores, ano de publicação, objetivo e atividades desenvolvidas.

Quadro 1 - Descrição dos artigos incluídos na revisão. Porto Alegre, RS, Brasil, 2015 (continua)

\begin{tabular}{|c|c|c|}
\hline $\begin{array}{l}\text { Autores e ano de } \\
\text { publicação }\end{array}$ & Objetivos & $\begin{array}{l}\text { Atividades desenvolvidas pelos } \\
\text { enfermeiros na transição do cuidado }\end{array}$ \\
\hline $\begin{array}{l}\text { Gunady S, Upfield } \\
\text { S, Pham ND, Yea J, } \\
\text { Schmiedeberg MB, } \\
\text { Stahmer GD. } 2015^{(14)}\end{array}$ & $\begin{array}{l}\text { Descrever o desenvolvimento e a } \\
\text { implementação de um programa de } \\
\text { transição do cuidado com foco na } \\
\text { reconciliação medicamentosa para } \\
\text { pacientes com insuficiência cardíaca. }\end{array}$ & Orientações sobre medicações. \\
\hline $\begin{array}{l}\text { Ulin K, Olsson LE, Wolf } \\
\text { A, Ekman I. 2015 }\end{array}$ & $\begin{array}{l}\text { Avaliar a eficiência do modelo de } \\
\text { planejamento de cuidados de um } \\
\text { Centro de Cuidados de Gotemburgo em } \\
\text { comparação com o cuidado usual em } \\
\text { pacientes com doença cardíaca. }\end{array}$ & $\begin{array}{l}\text { Elaboração do plano de alta, auxílio na } \\
\text { reabilitação social, orientações sobre } \\
\text { sinais e sintomas e autocuidado no } \\
\text { domicílio, articulação dos enfermeiros } \\
\text { do hospital com atenção primária à } \\
\text { saúde e visita domiciliar. }\end{array}$ \\
\hline $\begin{array}{l}\text { Young Young L, } \\
\text { Barnason S, Hays K, Do } \\
\text { V. } 2015^{(16)}\end{array}$ & $\begin{array}{l}\text { Avaliar os efeitos do gerenciamento } \\
\text { de cuidados na reconciliação } \\
\text { medicamentosa na alta de um hospital } \\
\text { rural para o domicilio. }\end{array}$ & Orientações sobre medicações. \\
\hline $\begin{array}{l}\text { Berry DL, Cunningham } \\
\text { T, Eisenberg } \\
\text { S, Wickline } \\
\text { M, Hammer M, Berg C. } \\
2014^{(17)}\end{array}$ & $\begin{array}{l}\text { Melhorar a compreensão do paciente } \\
\text { sobre suas medicações na alta hospitalar. }\end{array}$ & $\begin{array}{l}\text { Orientações sobre medicações e contato } \\
\text { telefônico de acompanhamento. }\end{array}$ \\
\hline $\begin{array}{l}\text { Biese K, Lamantia } \\
\text { M, Shofer F, McCall } \\
\text { B, Roberts E, Stearns } \\
\text { SC, et al. } 2014^{(18)}\end{array}$ & $\begin{array}{l}\text { Investigar se o acompanhamento } \\
\text { telefônico melhora a adesão de idosos } \\
\text { ao plano de cuidados após atendimento } \\
\text { em serviço de emergência e reduz } \\
\text { internações após } 35 \text { dias. }\end{array}$ & $\begin{array}{l}\text { Realização do planejamento de alta, } \\
\text { orientações sobre medicações, contato } \\
\text { telefônico de acompanhamento, visita } \\
\text { domiciliar. }\end{array}$ \\
\hline $\begin{array}{l}\text { Black JT, Romano } \\
\text { PS, Sadeghi } \\
\text { B, Auerbach } \\
\text { AD, Ganiats } \\
\text { TG, Greenfield S, et al. } \\
2014^{(19)}\end{array}$ & $\begin{array}{l}\text { Avaliar a eficácia de intervenção na } \\
\text { transição de cuidados com educação } \\
\text { pré-alta e telefonemas pós-alta. }\end{array}$ & $\begin{array}{l}\text { Realização do planejamento de alta, } \\
\text { orientações sobre medicações, contato } \\
\text { telefônico de acompanhamento, visita } \\
\text { domiciliar. }\end{array}$ \\
\hline $\begin{array}{l}\text { Englander H, Michaels } \\
\text { L, Chan B, Kansagara } \\
\text { D. } 2014^{(20)}\end{array}$ & $\begin{array}{l}\text { Avaliar o impacto de um programa de } \\
\text { transição do cuidado nas readmissões } \\
\text { em } 30 \text { dias, no uso de serviço de } \\
\text { emergência, na qualidade da transição e } \\
\text { na mortalidade. }\end{array}$ & $\begin{array}{l}\text { Elaboração do plano de alta, auxílio na } \\
\text { reabilitação social, orientações sobre } \\
\text { medicações, contato telefônico de } \\
\text { acompanhamento, visita domiciliar. }\end{array}$ \\
\hline
\end{tabular}




\begin{tabular}{|c|c|c|}
\hline $\begin{array}{l}\text { Harrison JD, Auerbach } \\
\text { AD, Quinn K, Kynoch } \\
\text { E, Mourad M. 2014 } \\
\end{array}$ & $\begin{array}{l}\text { Determinar o efeito de ligações } \\
\text { telefônicas pós-alta nas causas de } \\
\text { readmissões em } 30 \text { dias. }\end{array}$ & $\begin{array}{l}\text { Orientações sobre medicações, sinais } \\
\text { e sintomas e autocuidado no domicílio, } \\
\text { contato telefônico de acompanhamento. }\end{array}$ \\
\hline $\begin{array}{l}\text { Li J, Wang H, Xie } \\
\text { H, Mei G, Cai W, Ye J, } \\
\text { et al. } 2014^{(22)}\end{array}$ & $\begin{array}{l}\text { Avaliar a eficácia de telefonemas de } \\
\text { enfermeiros em pacientes com diálise } \\
\text { peritoneal na China continental. }\end{array}$ & $\begin{array}{l}\text { Elaboração do plano de alta, auxílio na } \\
\text { reabilitação social, orientações sobre } \\
\text { medicações e autocuidado no domicílio, } \\
\text { contato telefônico de acompanhamento. }\end{array}$ \\
\hline $\begin{array}{l}\text { Graham J, Gallagher } \\
\text { R, Bothe J. 2013 }\end{array}$ & $\begin{array}{l}\text { Verificar a compreensão dos enfermeiros } \\
\text { sobre planejamento de alta, adesão e } \\
\text { barreiras. }\end{array}$ & $\begin{array}{l}\text { Elaboração do plano de alta, auxílio na } \\
\text { reabilitação social, orientações para } \\
\text { autocuidado no domicílio. }\end{array}$ \\
\hline $\begin{array}{l}\text { Bradley EH, Curry L, } \\
\text { Horwitz LI, Sipsma H, } \\
\text { Wang Y, Walsh MN et } \\
\text { al. } 2013^{(24)}\end{array}$ & $\begin{array}{l}\text { Identificar estratégias adotadas pelos } \\
\text { hospitais para diminuir taxas de } \\
\text { readmissão de pacientes com infarto } \\
\text { agudo do miocárdio (IAM). }\end{array}$ & $\begin{array}{l}\text { Elaboração do plano de alta, orientações } \\
\text { sobre medicações. }\end{array}$ \\
\hline $\begin{array}{l}\text { Keeping-Burke } \\
\text { L, Purden M, Frasure- } \\
\text { Smith N, Cossette } \\
\text { S, McCarthy F, Amsel } \\
\text { R. } 2013^{(25)}\end{array}$ & $\begin{array}{l}\text { Identificar se um programa de telesaúde, } \\
\text { acompanhado de educação padronizada, } \\
\text { reduz ansiedade de cuidadores e } \\
\text { pacientes com necessidade de cirurgia } \\
\text { de revascularização coronariana. }\end{array}$ & $\begin{array}{l}\text { Realização do planejamento de alta, } \\
\text { auxílio na reabilitação social, orientações } \\
\text { sobre medicações, sinais e sintomas, } \\
\text { contato telefônico de acompanhamento, } \\
\text { visita domiciliar. } \\
\end{array}$ \\
\hline $\begin{array}{l}\text { Kind J, Jensen L, Barczi } \\
\text { S, Bridges A, Kordahl } \\
\text { R, Smith MA et al. } \\
2013^{(26)}\end{array}$ & $\begin{array}{l}\text { Avaliar um protocolo de transição do } \\
\text { cuidado para pacientes veteranos de } \\
\text { guerra após alta do hospital para o } \\
\text { domicílio. }\end{array}$ & $\begin{array}{l}\text { Orientações sobre medicações, sinais } \\
\text { e sintomas e autocuidado no domicílio, } \\
\text { articulação dos enfermeiros do hospital } \\
\text { com atenção primária à saúde, contato } \\
\text { telefônico de acompanhamento, visita } \\
\text { domiciliar. }\end{array}$ \\
\hline $\begin{array}{l}\text { Meisinger C, } \\
\text { Stollenwerk } \\
\text { B, Kirchberger I, Seidl } \\
\text { H, Wende R, Kuch B et } \\
\text { al. } 2013^{(27)}\end{array}$ & $\begin{array}{l}\text { ção de } \\
\text { idado na alta } \\
\text { com IAM. }\end{array}$ & $\begin{array}{l}\text { Auxílio na reabilitação social, orientações } \\
\text { sobre sinais e sintomas e autocuidado } \\
\text { no domicílio, contato telefônico de } \\
\text { acompanhamento, visita domiciliar. }\end{array}$ \\
\hline $\begin{array}{l}\text { Barnason } \\
\text { S, Zimmerman } \\
\text { L, Hertzog M, Schulz P. } \\
2010^{(28)}\end{array}$ & $\begin{array}{l}\text { Avaliar o impacto de uma intervenção de } \\
\text { transição do cuidado do hospital para o } \\
\text { domicílio em idosos pós-IAM em uso de } \\
\text { múltiplas medicações. }\end{array}$ & $\begin{array}{l}\text { Realização do planejamento de alta, } \\
\text { auxílio na reabilitação social, orientações } \\
\text { sobre alimentação, medicações, sinais } \\
\text { e sintomas, autocuidado no domicílio, } \\
\text { contato telefônico de acompanhamento. }\end{array}$ \\
\hline $\begin{array}{l}\text { CF, Setter SM, Daratha } \\
\text { KB, Neumiller JJ, Wood } \\
\text { LD. } 2010^{(29)}\end{array}$ & $\begin{array}{l}\text { Descrever as principais discrepâncias } \\
\text { medicamentosas identificadas pelas } \\
\text { enfermeiras na transição do cuidado de } \\
\text { adultos hospitalizados para o domicilio. }\end{array}$ & $\begin{array}{l}\text { Orientações sobre medicações, visita } \\
\text { domiciliar. }\end{array}$ \\
\hline $\begin{array}{l}\text { Portillo MC, Corchón } \\
\text { S, López-Dicastillo } \\
\text { O, Cowley S. } 2010^{(30)}\end{array}$ & $\begin{array}{l}\text { Avaliar a efetividade de um programa } \\
\text { de reabilitação social de pacientes } \\
\text { neurológicos e seus cuidadores. }\end{array}$ & $\begin{array}{l}\text { Realização do planejamento de alta, } \\
\text { auxílio na reabilitação social, sinais e } \\
\text { sintomas, autocuidado no domicílio. }\end{array}$ \\
\hline $\begin{array}{l}\text { Balaban RB, Weissman } \\
\text { JS, Samuel } \\
\text { PA, Woolhandler S. } \\
2008^{(31)}\end{array}$ & $\begin{array}{l}\text { Avaliar uma intervenção para reconectar } \\
\text { imediatamente pacientes nos cuidados } \\
\text { domiciliares após alta hospitalar. }\end{array}$ & $\begin{array}{l}\text { Elaboração do plano de alta, articulação } \\
\text { dos enfermeiros do hospital com atenção } \\
\text { primária à saúde, contato telefônico de } \\
\text { acompanhamento. }\end{array}$ \\
\hline $\begin{array}{l}\text { Setter SM, Corbett } \\
\text { CF, Neumiller JJ, Gates } \\
\text { BJ, Sclar DA, Sonnett } \\
\text { TE. 2009(32) }\end{array}$ & $\begin{array}{l}\text { Avaliar a colaboração de enfermeiros } \\
\text { na resolução de discrepâncias } \\
\text { medicamentosa em pacientes adultos e } \\
\text { idosos na transição do hospital para o } \\
\text { domicilio. }\end{array}$ & $\begin{array}{l}\text { Orientações sobre medicações, visita } \\
\text { domiciliar. }\end{array}$ \\
\hline $\begin{array}{l}\text { Chow SK, Wong } \\
\text { FK, Chan TM, Chung } \\
\text { LY, Chang KK, Lee RP. } \\
2007^{(33)}\end{array}$ & $\begin{array}{l}\text { Avaliar os serviços de enfermeiros } \\
\text { comunitários para pacientes com } \\
\text { doenças crônicas na transição do } \\
\text { hospital para o domicilio. }\end{array}$ & $\begin{array}{l}\text { Elaboração do plano de alta, auxílio na } \\
\text { reabilitação social, orientações sobre } \\
\text { alimentação, medicações e sinais e } \\
\text { sintomas, visita domiciliar. } \\
\end{array}$ \\
\hline Tarling; Jauffur. 2006 & $\begin{array}{l}\text { Produzir protocolo de intervenção } \\
\text { baseado em melhores práticas para } \\
\text { padronizar plano de alta multidisciplinar. }\end{array}$ & $\begin{array}{l}\text { Elaboração do plano de alta, auxílio na } \\
\text { reabilitação social. }\end{array}$ \\
\hline
\end{tabular}




\begin{tabular}{|l|l|l|}
$\begin{array}{l}\text { Wong FK, Mok } \\
\text { MP, Chan T, Tsang } \\
\text { MW. } 2005^{(35)}\end{array}$ & $\begin{array}{l}\text { Comparar resultados obtidos no } \\
\text { acompanhamento por telefone de } \\
\text { pacientes com alta precoce com os que } \\
\text { receberam orientações de rotina na alta } \\
\text { hospitalar. }\end{array}$ & $\begin{array}{l}\text { Orientações sobre alimentação, } \\
\text { medicações, sinais e sintomas, } \\
\text { autocuidado no domicílio, contato } \\
\text { telefônico de acompanhamento. }\end{array}$ \\
\hline
\end{tabular}

Quanto ao delineamento metodológico das publicações, destaca-se elevado número de estudos relacionados a intervenções. Foram identificados oito (36,4\%) ensaios clínicos randomizados, três $(13,6 \%)$ estudos quase-experimentais do tipo antes-depois, dois $(9,1 \%)$ protocolos de intervenção, um $(4,5 \%)$ ensaio clínico não randomizado, um $(4,5 \%)$ estudo piloto de intervenção e um $(4,5 \%)$ pesquisa ação. Demais delineamentos encontrados foram quatro $(18,3 \%)$ estudos quantitativos descritivos e dois $(9,1 \%)$ qualitativos exploratórios.

Em relação ao ano de publicação, em 2005, 2006, 2007 e 2008 houve publicação de um artigo por ano. Foram publicados três artigos em 2010, cinco em 2013, seis em 2014 e três em 2015. Identificouse predominância de estudos com foco em idosos e pessoas com doenças crônicas, como diabetes, doenças cardiovasculares, pulmonares, câncer e neurológicas.

As atividades dos enfermeiros descritas nos 22 artigos foram classificadas em cinco categorias temáticas: Planejamento de cuidados para a alta; Auxílio na reabilitação social; Educação em saúde; Articulação com os demais serviços; Acompanhamento pós-alta.

\section{Categoria 1 - Planejamento de cuidados para alta}

Nesta categoria foram incluídas atividades descritas em 13 artigos que compuseram a amostra. O planejamento de alta foi realizado pelo enfermeiro hospitalar ${ }^{(18-19,22-23,28,31)}$, em conjunto com o paciente e familiares ${ }^{(25)}$ ou com equipe multiprofissional ${ }^{(15,20,32,35)}$ e reformulado durante a internação, de acordo com mudanças clínicas e psicossociais do paciente ${ }^{(15)}$. Os planos de alta continham informações sobre diagnósticos prévios ${ }^{(24-25)}$, modo de administração das medicações ${ }^{(18,20,24-25,31,33)}$, história pregressa, avaliação da condição psicossocial(20,22-23,25,33), condições financeiras e de moradia ${ }^{(31)}$ e acompanhamento após a alta ${ }^{(24-25,31,33)}$. O plano foi entregue ao paciente no momento da alta hospitalar ${ }^{(15)}$.

\section{Categoria 2 - Auxílio na Reabilitação Social}

As atividades incluídas nesta categoria foram identificadas em 10 artigos. A reabilitação social visa a retomada da vida cotidiana do paciente após a alta hospitalar ${ }^{(30)}$. É centrada na interação entre família e comunidade, promoção de atitudes positivas, aceitação e adaptação da doença nas atividades de vida diárias, diminuindo a sensação de abandono, propiciando expectativas favoráveis à reabilitação ${ }^{(15,30)}$. Os enfermeiros avaliaram aspectos que podiam auxiliar ou dificultar a recuperação, como condições físicas $^{(22,27,30,33)}$, sociais ${ }^{(15,22,33,34)}$, psicológicas $^{(20,22-23,25,28,30,33)}$ e motivacionais ${ }^{(28)}$ do paciente e família.

\section{Categoria 3 - Educação em Saúde}

Essa atividade foi encontrada em 20 artigos, sendo a predominante nos estudos identificados nesta revisão. Os enfermeiros realizam educação em saúde sob diversos aspectos: mudança na dieta e possíveis restrições de alimentos ${ }^{(27-28,33,35)}$, realização de exercícios físicos ${ }^{(25,35)}$, uso correto das medicações, como dosagem, frequência de administração e horários ${ }^{(14,17-19,21-22,25-26,22-30,33)}$, interações dos medicamentos de uso contínuo ${ }^{(14,16,20,26,32,35)}$, reconhecimento de sinais e sintomas da doença em curso $^{(19-20,22-23,25-28,30,35)}$ e autocuidado no domicílio ${ }^{(15,22,35)}$. Alguns enfermeiros realizam a reconciliação medicamentosa, avaliando medicamentos de uso anterior à internação com os prescritos no ambiente hospitalar ${ }^{(16,20,24)}$. Outros utilizam apoio de encartes ilustrativos para reforçar orientações dos cuidados $^{(25,26,28)}$ e linguagem simples, além da utilização do feedback das informações para verificar a compreensão do paciente quanto às informações prestadas ${ }^{(19,30)}$. 


\section{Categoria 4 - Articulação com os demais serviços}

Essa atividade foi descrita em três estudos da amostra. Os enfermeiros transmitem informações sobre o plano de alta do paciente para as equipes da atenção primária à saúde, gerenciando o processo de transição do cuidado entre diferentes pontos da rede de atenção à saúde. Um dos modos de articulação entre serviços é feito por meio de notificações dos enfermeiros hospitalares sobre a alta do paciente ao serviço de referência ${ }^{(15)}$.

Outro modo de articulação entre serviços é feito por meio de telefonemas dos enfermeiros hospitalares, para que o enfermeiro da atenção primária realize visita domiciliar e esclareça as dúvidas do paciente ${ }^{(26)}$. Também é utilizado o sistema informatizado do hospital, interligado à rede de saúde local. O plano de alta é enviado eletronicamente para o serviço de atenção primária e utilizado pela equipe de saúde para acompanhamento dos cuidados domiciliares ${ }^{(31)}$.

\section{Categoria 5 - Acompanhamento pós-alta}

As atividades dos enfermeiros de acompanhamento pós-alta foram descritas em 16 artigos. Foram realizadas ligações telefônicas ou visitas domiciliares, com a finalidade de avaliar o plano de alta e abordar as orientações prestadas no ambiente hospitalar e esclarecer possíveis dúvidas e dificuldades quanto a essas informações ${ }^{(21,33)}$. Os pontos principais são: identificar e orientar aspectos do tratamento ${ }^{(18,27-28,31)}$, sinais e sintomas de alarme ${ }^{(25,33,35)}$, administrações dos medicamentos ${ }^{(17,25-26)}$, verificar a compreensão das atividades de autogerenciamento dos cuidados ${ }^{(20-21,33)}$, elucidar quanto aos locais adequados para atendimento $^{(33)}$ e consultas de acompanhamento ${ }^{(25,27,31)}$.

Contatos telefônicos permitiram a identificação de dúvidas de pacientes e cuidadores no contexto domiciliar ${ }^{(18-19,22)}$, definição de visitas domiciliares aos pacientes de risco ${ }^{(20)}$, e intervenções imediatas durante a ligação ${ }^{(31)}$.

\section{DISCUSSÃO}

Identifica-se que as atividades dos enfermeiros na transição do cuidado na alta do hospital para o domicílio são múltiplas e realizadas em diferentes complexidades, iniciando-se no período da internação e completando-se quando o paciente se insere no ambiente domiciliar.

Os planejamentos dos cuidados de alta iniciados na admissão estabelecem objetivos para tratamento a curto e em longo prazo, além de estimular o vínculo e participação de pacientes e familiares nas escolhas de cuidado(2). A elaboração de metas para o tratamento fortaleceu os cuidados de acordo com as necessidades de saúde, propiciando recuperação segura e efetiva ${ }^{(15)}$. Planejamentos de alta compartilhados possibilitam agilidade no trabalho do enfermeiro e da equipe, favorecendo pacientes e famílias. A coleta de informações pelos profissionais de saúde esclarece o motivo da internação e possíveis dificuldades do tratamento no ambiente domiciliar, gerenciando cuidados individualizados na internação e promovendo a saúde no pós-alta.

Em um ambiente de cuidado continuado, é importante acompanhar a adaptação do paciente ${ }^{(5)}$ e sua interação com a família, fundamentais para a retomada das atividades diárias ${ }^{(30)}$. Para tanto, enfermeiros podem avaliar condições físicas e psicossociais dos pacientes e promover assistência de qualidade aos vulneráveis para que mantenham suas atividades convivendo com a doença ${ }^{(22,28)}$. Essa avaliação fornece subsídios para promover a reabilitação do paciente, a fim de buscar sua integração com a família e a comunidade da qual faz parte.

Os profissionais devem avaliar a facilidade do paciente para aceitar mudanças e identificar aspectos psicológicos e cognitivos que possam orientar a compreensão dos cuidados ${ }^{(23)}$. Programas de auxílio na reabilitação social mostram-se valiosos para transições do cuidado efetivas e de qualidade, especialmente aos pacientes mais vulneráveis, possibilitando sensação de segurança, adaptação à doença e às atividades no domicílio ${ }^{(6)}$.

A educação em saúde é a atividade que se destaca dentre os estudos. Assim como a reabilitação 
social e o planejamento de alta, pressupõe a participação dos pacientes na construção dos seus cuidados a partir de informações compartilhadas.

Estratégias de educação desenvolvidas utilizando comunicação clara e objetiva, uso de linguagem simples, encartes ilustrativos e feedback de informações contribuem com o entendimento dos cuidados pelos pacientes e familiares ${ }^{(5-6,11)}$. As orientações disponibilizadas pelos enfermeiros mostramse essenciais para efetivas transições, pois favorecem o uso das medicações e o gerenciamento do autocuidado ${ }^{(17)}$, aumentam a adesão ao tratamento ${ }^{(35)}$, reduzem a taxa de reinternação(15,21,26-27) e de mortalidade $^{(20,27)}$.

O número de investigações com foco na educação em saúde indica preocupação com a inclusão dos pacientes e famílias no cuidado, de forma a melhorar aspectos da alimentação, execução de atividade física, uso correto das medicações e reconhecimento precoce de sinais e sintomas. Há que se pensar no cuidado integral e individualizado, ressaltando-se que a educação em saúde não pode ser apenas prescritiva, mas também esclarecedora. Sendo assim, investir em ações de autocuidado e apoio no gerenciamento de medicações empoderam pacientes e cuidadores, evitando procura e uso inapropriado dos serviços disponíveis na rede atenção à saúde.

O acompanhamento pós-alta é necessário para identificar dúvidas sobre o tratamento prescrito no hospital e saber onde buscar assistência caso surja problema inesperado ${ }^{(25)}$. Esse propósito foi implementado pelo acompanhamento por telefone, pela visita domiciliar e agendamento de consultas após a alta, com obtenção de resultados positivos ${ }^{(26,31)}$.

A articulação do hospital com os demais serviços da rede assistencial ainda é uma atividade pouco realizada, demonstrando que embora haja preocupação com o acompanhamento pós-alta, esta atividade é feita pelo serviço de atenção primária. Três estudos ${ }^{(15,26,31)}$ utilizaram a articulação entre serviços, enfatizando que a comunicação entre diferentes níveis de atenção auxiliou na implementação de melhores práticas de transição e possibilitou continuidade dos cuidados pós-alta.

Processos de transições de maior qualidade pressupõem foco nas necessidades individuais do paciente e família e no cuidado compartilhado ${ }^{(5,9,15)}$. Dessa forma, profissionais, pacientes e familiares tornam-se parceiros no cuidado desde o momento de internação até os dias subsequentes ao regresso ao domicílio.

As atividades dos enfermeiros para desenvolver a coordenação dos cuidados na transição do hospital para o domicílio incluem reconciliação medicamentosa, orientação ao paciente e/ou cuidador, seguimento domiciliar do paciente após alta hospitalar, comunicação efetiva entre hospital e demais serviços de saúde, e apoio na comunidade. No entanto, há que se ampliar e valorizar sua atuação e qualificar suas atividades, em busca de transições cada vez mais eficazes.

\section{- CONCLUSÃO}

Os resultados deste estudo permitiram identificar as principais atividades dos enfermeiros na transição do cuidado. Identificou-se o aumento do número de artigos no decorrer dos últimos cinco anos, o que demonstra a crescente relevância da temática. A maioria dos estudos incluídos nesta revisão eram experimentais e quase-experimentais, sugerindo interesse na melhoria da efetividade e da qualidade das transições do cuidado, especialmente nas condições crônicas de maior vulnerabilidade.

Salienta-se que há muitos estudos com múltiplas intervenções que analisam os efeitos das iniciativas lideradas pelas enfermeiras, porém os resultados não elucidam claramente quais aspectos da intervenção foram responsáveis pelo efeito favorável.

Aspectos relacionados à preparação dos cuidados para alta, educação em saúde de pacientes e familiares, apoio na continuidade dos cuidados no domicílio e acompanhamento pós-alta são indicadores para avaliação da qualidade do atendimento prestado nos serviços de saúde. Assim, este estudo fornece informações para aprimoramento das práticas assistenciais e organização das atividades dos enfermeiros, para promover a coordenação dos cuidados para altas hospitalares com foco na transição do cuidado. 
Diante dos achados, salienta-se a falta de estudos sobre as atividades na rotina da prática assistencial dos enfermeiros na transição do cuidado. É uma temática que necessita de mais pesquisas no Brasil, pois a transição do cuidado é uma estratégia que pode contribuir para a efetivação de sistemas integrados de saúde.

\section{- REFERÊNCIAS}

1. Coleman EA, Boult C, American Geriatrics Society Health Care Systems Committee. Improving the quality of transitional care for persons with complex care needs. J Am Geriatr Soc. 2003;51(4):556-7.

2. Hesselink G, Flink M, Olsson M, Barach P, Dudzik-Urbaniak E, Orrego C, et al. Are patients discharged with care? A qualitative study of perceptions and experiences of patients, family members and care providers. BMJ Qual Saf. [Internet] 2012;21(Suppl 1) [acesso em 1 jul 2016]. Disponível: http://dx.doi.org/10.1136/bmjqs-2012-001165.

3. Meyers AG, Salanitro A, Wallston KA, Cawthon C, Vasilevskis EE, Goggins KM, et al. Determinants of health after hospital discharge: rationale and design of the Vanderbilt Inpatient Cohort Study (VICS). BMC Health Serv Res. [Internet] 2014;(14) [acesso em 1 jul 2016]. Disponível: http://dx.doi.org/10.1186/1472-6963-14-10.

4. Delatorre PG, Sá SPC, Valente GSC, Silvino ZR. Planejamento para a alta hospitalar como estratégia de cuidado de enfermagem: revisão integrativa. Rev enferm UFPE on line. [Internet] 2013;7(12) [acesso em 4 jul 2016]. Disponível: http://www.revista.ufpe.br/revistaenfermagem/index.php/revista/article/view/3968/pdf_4295.

5. Guerrero KS, Puls SE, Andrew DA. Transition of care and the impact on the environment of care. J Nurs Educ Pract. 2014;4(6):30-6.

6. Coffey A, Mccarthy GM. Older people's perception of their readiness for discharge and post discharge use of community support and services. Int J Older People Nurs. 2013;8(2):104-15.

7. Miller JF, Piacentine LB, Weiss, M. Coping difficulties after hospitalization. Clin Nurs Res. 2008;17(4):278-96.

8. Trompeter JM, McMillan AN, Rager ML, Fox JR. Medication Discrepancies during Transitions of Care: A Comparison Study. J Healthc Qual. 2015;37(6):325-32.

9. Couzner L, Ratcliffe J, Crotty M. The relationship between quality of life, health and care transition: an empirical comparison in an older post-acute population. Health Qual Life Outcomes. [Internet] 2012;(10) [acesso em 4 jul 2016]. Disponível: http://dx.doi.org/10.1186/1477-7525-10-69.

10. Flemming MO, Haney TT. Improving patient outcomes with better care transitions: the role for home health. Cleve Clin J Med. [Internet] 2013;80(Suppl 1) [acesso em 4 jul 2016]. Disponível: http://dx.doi.org/10.3949/ ccjm.80.e-s1.02.

11. Dusek B, Pearce N, Harripaul A, Lloyd M. Care transitions a systematic review of best practices. J Nurs Care Qual. 2015;30(3):233-9.

12. Romagnoli KM, Handler SM, Ligons FM, Hochheiser H. Home-Care Nurses' perceptions of Unmet Information Needs and Communication Difficulties of Geriatric Patients in the Immediate Post-hospital Discharge Period. BMJ Qual Saf. 2013;22(4):324-32.

13. Whittemore R, Knafl K. The integrative review: updates methodology. J Adv Nurs. 2005;52(5):546-53.

14. Gunadi S, Upfield S, Pham ND, Yea J, Schmiedeberg MB, Stahmer GD. Development of a collaborative transitions-of-care program for heart failure patients. Am J Health-Syst Pharm. 2015;72(13):1147-52.

15. Ulin K, Olsson LE, Wolf A, Ekman I. Person-centred care - An approach that improves the discharge process. Eur J Cardiovasc Nurs. [Internet] 2015;15(3) [acesso em 4 jul 2016]. ahead of print Epub 1 jul 2016. Disponível: http://dx.doi.org/10.1177/1474515115569945.

16. Young L, Barnason S, Hays K, Do V. Nurse Practitioner - led medication reconciliation in critical access hospitals. J Nurse Pract. 2015;11(5):511-8. 
17. Berry DL, Cunningham T, Eisenberg S, Wickline M, Hammer M, Berg C. Improving patient knowledge of discharge medications in an oncology setting. Clin J Oncol Nurs. 2014;18(1):35-7.

18. Biese K, Lamantia M, Shofer F, McCall B, Roberts E, Stearns SC, et al. A randomized trial exploring the effect of a telephone call follow-up on care plan compliance among older adults discharged home from the emergency department. Acad Emerg Med. 2014;21(2):188-95.

19. Black JT, Romano PS, Sadeghi B, Auerbach AD, Ganiats TG, Greenfield S, et al. A remote monitoring and telephone nurse coaching intervention to reduce readmissions among patients with heart failure: study protocol for the Better Effectiveness After Transition - Heart Failure (BEAT-HF) randomized controlled trial. Trials. [Internet] 2014;(15) [acesso em 4 jul 2016]. Disponível: http://dx.doi.org/10.1186/1745-6215-15-124.

20. Englander H, Michaels L, Chan B, Kansagara D. The care transitions innovation (C-Traln) for socioeconomically disadvantaged adults: results of a cluster randomized controlled trial. J Gen Intern Med. 2014;29(11):1460-7.

21. Harrison JD, Auerbach AD, Quinn K, Kynoch E, Mourad M. Assessing the impact of nurse post-discharge telephone calls on 30-day hospital readmission rates. J Gen Intern Med. 2014;29(11):1519-25.

22. Li J, Wang H, Xie H, Mei G, Cai W, Ye J, et al. Effects of post-discharge nurse-led telephone supportive care for patients with chronic kidney disease undergoing peritoneal dialysis in china: a randomized controlled trial. Perit Dial Int. 2014;34(3):278-88.

23. Graham J, Gallagher R, Bothe J. Nurses' discharge planning and risk assessment: behaviours, understanding and barriers. J Clin Nurs. 2013;22(15-16):2338-46.

24. Bradley EH, Curry L, Horwitz LI, Sipsma H, Wang Y, Walsh MN, et al. Hospital Strategies Associated With 30Day Readmission Rates for Patients With Heart Failure. Circ Cardiovasc Qual Outcomes. 2013;6(4):444-50.

25. Keeping-Burke L, Purden M, Frasure-Smith N, Cossette S, McCarthy F, Amsel R. Bridging the transition from hospital to home: effects of the VITAL telehealth program on recovery for CABG surgery patients and their caregivers. Res Nurs Health. 2013;36(6):540-53.

26. Kind J, Jensen L, Barczi S, Bridges A, KordahI R, Smith MA, et al. Low-cost transitional care with nurse managers making mostly phone contact with patients cut rehospitalization at a VA hospital. Health Aff (Millwood). 2012;31(12):2659-68.

27. Meisinger C, Stollenwerk B, Kirchberger I, Seidl H, Wende R, Kuch B, et al. Effects of a nurse-based case management compared to usual care among aged patients with myocardial infarction: results from the randomized controlled KORINNA study. BMC Geriatr. [Internet] 2013;(13) [acesso em 4 jul 2016]. Disponível: http://dx.doi.org/10.1186/1471-2318-13-115.

28. Barnason S, Zimmerman L, Hertzog M, Schulz P. Pilot testing of a medication self- management transition intervention for heart failure patients. West J Nurs Res. 2010;32(7):849-70.

29. Corbett CF, Setter SM, Daratha KB, Neumiller JJ, Wood LD. Nurse identified hospital to home medication discrepancies: implications for improving transitional care. Geriatr Nurs. 2010;31(3):188-96.

30. Portillo MC, Corchón S, López-Dicastillo O, Cowley S. Evaluation of a nurse-led social rehabilitation programme for neurological patients and carers: An action research study. Int J Nurs Stud. 2009;46(2):204-19.

31. Balaban RB, Weissman JS, Samuel PA, Woolhandler S. Redefining and redesigning hospital discharge to enhance patient care: a randomized controlled study. J Gen Intern Med. 2008;23(8):1228-33.

32. Setter SM, Corbett CF, Neumiller JJ, Gates BJ, Sclar DA, Sonnett TE. Effectiveness of a pharmacist-nurse intervention on resolving medication discrepancies for patients transitioning from hospital to home health care. Am J Health Syst Pharm. 2009;66(22):2027-31.

33. Chow SK, Wong FK, Chan TM, Chung LY, Chang KK, Lee RP. Community nursing services for post discharge chronically ill patients. J Clin Nurs. 2008;17(7B):260-71.

34. Tarling M, Jauffur H. Improving team meetings to support discharge planning. Nurs Times. 2006;102(26):32-5. 
Cogitare Enferm. (22)3: e47615, 2017

35. Wong FK, Mok MP, Chan T, Tsang MW. Nurse follow-up of patients with diabetes: randomized controlled Trial. J Adv Nurs. 2005;50(4):391-402. 